\title{
Experiential Avoidance and Rumination in Parents of Children on Cancer Treatment: Relationships with Posttraumatic Stress Symptoms and Symptoms of Depression
}

\author{
Martin Cernvall $^{1} \cdot$ Ellen Skogseid $^{1} \cdot$ Per Carlbring $^{2} \cdot$ Lisa Ljungman $^{1} \cdot$ \\ Gustaf Ljungman $^{3} \cdot$ Louise von Essen $^{1}$
}

Published online: 13 October 2015

(c) The Author(s) 2015. This article is published with open access at Springerlink.com

\begin{abstract}
We conducted a cross-sectional survey study to investigate whether there is a relationship between experiential avoidance (EA), rumination, post-traumatic stress symptoms (PTSS), and symptoms of depression, in parents of children on cancer treatment. Data from 79 parents $(55$ mothers) of 79 children with a median of three months since their cancer diagnosis were included in cross-sectional analyses. EA and rumination were positively correlated with PTSS and symptoms of depression. EA and rumination did not provide incremental explained variance in PTSS over and above that explained by symptoms of depression, while controlling for symptoms of anxiety and demographic characteristics. However, EA and rumination provided incremental explained variance in symptoms of depression over and above that explained by PTSS, while controlling for symptoms of anxiety and demographic characteristics. Rumination and EA are important constructs in the understanding of PTSS and symptoms of depression in parents of children on cancer treatment. Future research should delineate the temporal relationships between these constructs.
\end{abstract}

Keywords Avoidance - Cancer and oncology . Depression · Parents · Posttraumatic stress · Rumination

Martin Cernvall

martin.cernvall@pubcare.uu.se

1 Clinical Psychology in Healthcare, Department of Public Health and Caring Sciences, Uppsala University, Box 564, 75122 Uppsala, Sweden

2 Department of Psychology, Stockholm University, Stockholm, Sweden

3 Pediatric Oncology, Department of Women's and Children's Health, Uppsala University, Uppsala, Sweden

\section{Introduction}

Symptoms of posttraumatic stress (PTSS) and depression are common among parents of children on cancer treatment (Boman, Viksten, Kogner, \& Samuelsson, 2004; Kazak, Boeving, Alderfer, Hwang, \& Reilly, 2005; Pöder, Ljungman, \& von Essen, 2008). Typically the level of such symptoms is higher shortly after diagnosis and decline with time after diagnosis (Ljungman, Hovén, Ljungman, Cernvall, \& von Essen, 2015). However, there is evidence for distinct subgroups with different development trajectories (Dolgin et al. 2007), and clinically relevant symptoms can continue for years after end of treatment (Ljungman et al., 2014). Despite this, there is a paucity of conceptual work on how to understand PTSS and symptoms of depression among parents of children on cancer treatment, especially concepts that can inform interventions. This study's purpose was to investigate the validity of a recent conceptualization in which the constructs of experiential avoidance (EA) and rumination are important components in maintaining PTSS and general psychological distress such as depression, in parents of children on cancer treatment (Cernvall, Carlbring, Ljungman, \& von Essen, 2013). This conceptualization is based on principles from cognitive and behavioral therapies (e.g., Farmer \& Chapman, 2008; Hayes, Strosahl, \& Wilson, 1999; Wells, 2008), and from the nature of PTSS and posttraumatic stress disorder (PTSD) as defined in current diagnostic manuals (e.g., American Psychiatric Association, 2000, 2013). Like many other psychiatric disorders, PTSD, and the closely related concept of PTSS, are not unidimensional concepts. Rather, PTSS and PTSD are psychiatric conglomerates, i.e., a diverse collection of cognitive, emotional, and behavioral components that occur together that have been identified as a psychiatric disorder. Thus, in the present study, the 
parents of a child with cancer can be seen to display some of the diverse behaviors grouped under the PTSS/PTSD label: having recurrent thoughts about events related to their child's disease; a tendency to avoid reminders of such events; and being in a state of hyper-arousal, all of which are understandable consequences of the perceived threat to their child's life and physical integrity.

Our approach assumes that it is essential to disaggregate the PTSS/PTSD conglomerate into more basic cognitive, motivational, and behavioral components, and to recognize that some of these components are fundamental psychological processes that contribute not only to PTSS and PTSD, but also to many other patterns of thought and behavior. We posit that EA and rumination are components of the PTSD/PTSS conglomerate, but that they are also integral parts of other clusters of human behavior, and so are more fundamental features of human behavior than the PTSS/PTSD conglomerate they influence. Thus, we view EA and rumination as separate and distinct entities in relation to the PTSS/PTSD conglomerate. In general, humans probably have an inborn and learned capacity to adapt to stressful events. However, some engage in behavioral processes that interfere with adaptation, which can maintain PTSS and increase general psychological distress. We hypothesize that EA and rumination are processes that interfere with adaptation to the stressful event of having a child on cancer treatment.

EA has been defined as "the phenomenon that occurs when a person is unwilling to remain in contact with private experiences (e.g., bodily sensations, emotions, thoughts, memories, behavioral predispositions) and takes steps to alter the form and frequency of these events and the contexts that occasion them" (Hayes, Wilson, Gifford, Follette, \& Strosahl, 1996, p. 1154). The suggested process is that such avoidance of inner experiences has a paradoxical effect, namely, an increase in the phenomena (e.g., thought or feeling) that the individual is trying to get rid of (Campbell-Sills, Barlow, Brown, \& Hofmann, 2006; Wenzlaff \& Wegner, 2000). In addition, avoidance increases physiological reactivity (Gross \& Levenson, 1997) and narrows behavioral repertoires, which results in psychological inflexibility that can hamper the individual's resources when coping with stressful situations, and so interferes with the ability to engage in behaviors with valued consequences. EA has been operationalized with the Acceptance and Action Questionnaire (AAQ) and its successor, the AAQ-II (Bond et al., 2011), which view EA as a psychological process underlying many forms of psychological distress and difficulties to adjust. EA is a risk factor for emotional disorder in healthy individuals and individuals with a prior history of emotional disorders (Spinhoven, Drost, de Rooij, van Hemert, \& Penninx, 2014) and is associated with PTSS among trauma survivors (Marx \&
Sloan, 2005; Orcutt, Pickett, \& Pope, 2005). Furthermore, EA is more strongly related to general distress and depression than is PTSS, and contributes to general distress and depression when PTSS is controlled for among undergraduates exposed to trauma and treatment seeking individuals (Plumb, Orsillo, \& Luterek, 2004; Tull, Gratz, Salters, \& Roemer, 2004). Thus, individuals who engage in EA subsequent to a stressful event are at risk for impaired psychological functioning (Fledderus, Bohlmeijer, \& Pieterse, 2010).

Rumination, i.e., excessive conceptual processing characterized by "long chains of predominantly verbal thought in which the person attempts to answer 'What if...?' questions or questions about the meaning of events, e.g., 'Why do I feel this way?'" (Wells, 2008, p. 11), is related to anxiety and depression. Rumination has been conceptualized as an emotion-regulating strategy characterized by avoidance of negative emotion and driven by meta-cognitive beliefs about its efficacy in remediating perceived discrepancies (Smith \& Alloy, 2009). Trauma-related rumination predicts depression and PTSS among roadtraffic accident survivors (Ehlers, Mayou, \& Bryant, 1998; Ehring, Frank, \& Ehlers, 2008; Murray, Ehlers, \& Mayou, 2002), and has been operationalized with one of the subscales in the Responses to Intrusions Questionnaire (Clohessy \& Ehlers, 1999; Murray et al., 2002).

Important to note are the conceptual differences between EA, rumination, and the avoidance symptoms characterizing PTSD. Avoidance in PTSD is focused on the experiences associated with a trauma (American Psychiatric Association, 2000). Rumination is the tendency to engage in repetitive and abstract thinking in response to intrusive thoughts about a trauma (Clohessy \& Ehlers, 1999). EA, on the other hand, is the tendency to avoid particular private experiences, which may or may not be associated with a trauma (Hayes et al., 1996). Taken together, EA is a broader construct and a behavioral tendency that may include rumination and avoidance symptoms characterizing PTSD.

Taken together, EA and rumination may interfere with psychological recovery after a trauma. Many parents of children on cancer treatment experience repeated and ongoing traumas such as receiving the child's diagnosis, a poor prognosis, invasive procedures, severe side-effects of treatments, and/or a recurrence. PTSS may be understandable reactions given such circumstances. However, based on the literature reviewed above, parents who engage in EA and/or trauma-related rumination in response to PTSS may be at risk for sub-optimal adaptation. To the best of our knowledge, the role of EA and rumination and their relationship with psychological distress among parents of children on cancer treatment is yet to be investigated. Such research could be of theoretical as well as 
clinical value since EA and rumination are modifiable constructs and interventions designed to target these processes do exist (Hayes et al., 1999; Wells, 2008). Knowledge about whether, and if so how, EA and/or rumination contribute to PTSS and depression in parents of children on cancer treatment could inform development of interventions for this population.

The study's purpose was to investigate whether there is a relationship between EA, rumination, PTSS, and symptoms of depression in parents of children on cancer treatment. Symptoms of anxiety were assumed to be important in this relationship and were investigated as a potential covariate. It was hypothesized that EA and rumination would be positively associated with symptoms of anxiety, PTSS, and symptoms of depression, and that EA and rumination would account for unique variance in PTSS and symptoms of depression when controlling for symptoms of anxiety and demographic characteristics. Based on earlier findings (Plumb et al., 2004; Tull et al., 2004), it was hypothesized that EA's contribution of unique variance to PTSS, when controlling for symptoms of depression, would be less pronounced than EA's contribution of unique variance to symptoms of depression when controlling for PTSS.

\section{Methods}

\section{Procedure and Participants}

Data were collected at the screening/pre-assessment of a randomized controlled trial (RCT) investigating the efficacy of Internet-based guided self-help for parents of children on cancer treatment (Cernvall, Carlbring, Ljungman, Ljungman, \& von Essen, 2015). Swedish speaking parents of children on treatment for any type of cancer disease with access to a computer with an Internet connection were potential participants. To be included in the RCT, participants had to meet the modified symptom criteria on the PTSD-Checklist Civilian Version (PCL-C; Weathers, Litz, Herman, Huska, \& Keane, 1993), a selfreport instrument corresponding to the DSM-IV model of PTSD (American Psychiatric Association, 2000), and not suffer from a psychiatric disorder in immediate need for treatment. The modified symptom criteria constitutes scoring $\geq 3$ on at least 1 of 5 symptoms of re-experiencing, 1 of 7 symptoms of avoidance, and 1 of 5 symptoms of hyper-arousal, corresponding to partial PTSD (Breslau, Lucia, \& Davis, 2004). All parents participating in the screening/pre-assessment were eligible for the current study. Potential participants were approached at five of the six Swedish pediatric oncology centers and asked to participate by a nurse or physician
4-12 weeks after their child's diagnosis. In the initial protocol potential participants were to be approached 1-2 weeks after diagnosis. However, during the first months of recruitment, it became evident that, for administrative reasons, parents were being approached considerably later than 1-2 weeks after diagnosis, and so the protocol for approaching parents was changed to 4-12 weeks after diagnosis. Participants answered questionnaires via the Internet. The procedure was approved by the regional ethics review board in Uppsala and all participants provided written informed consent. As shown in Fig. 1, $26 \%$ (194 of 747) of those informed about the study consented to be contacted. Due to ethical restrictions data were not collected from those declining participation, precluding conclusions regarding whether these individuals differed from those consenting, or their reasons for declining. However, common reasons, as reported by staff that approached parents, were not having the need and/or not having the time to participate. One-hundred parents, $52 \%$ of those who consented to be contacted, provided complete assessments. As some were nested in the same child, and to avoid dependency among observations (Kenny, 2011), data from 21 parents who completed the assessment were not included in the analyses. In case there were two parents, data from the parent who first provided data were included.

The sample included in the analyses consisted of 79 parents, 55 mothers $(70 \%)$ and 24 fathers $(30 \%)$ of 79 children, 45 girls $(57 \%)$ and 34 boys $(43 \%)$. The 21 parents for whom data were not included in the analyses did not differ from those whose data were included on demographic characteristics or study variables except for time since diagnosis for which excluded parents completed the assessment later (median $=4$ months, interquartile range $[\mathrm{IQR}]=3$ ) compared to included parents (median $=3$ months, IQR $=1), p<.05$. The parents' mean age was 39 years $(\mathrm{SD}=7.2, n=76$ due to missing data for three participants). All participants were biological parents to the children on cancer treatment, and 62 (78\%) were living with the sick child's other biological parent, seven $(9 \%)$ were living with a stepparent to the sick child and $10(13 \%)$ were living alone with the child. Thirtyseven $(47 \%)$ had finished university education, 37 (47\%) had finished upper secondary school and five $(6 \%)$ had finished elementary school. Sixty-seven $(85 \%)$ were employed, six $(8 \%)$ were unemployed and six $(8 \%)$ were on sick-leave. Thirty-seven (47\%) reported having experienced at least one previous trauma. The median distance from the pediatric oncology center where the child received its care was $48 \mathrm{~km}(\mathrm{IQR}=95, n=77$ due to missing data for two participants). At the time of assessment, the children's mean age was 8.0 years $(\mathrm{SD}=5.4), 42(53 \%)$ were diagnosed with leukemia, $11(14 \%)$ with sarcoma, six 
Fig. 1 Participant flow through the study

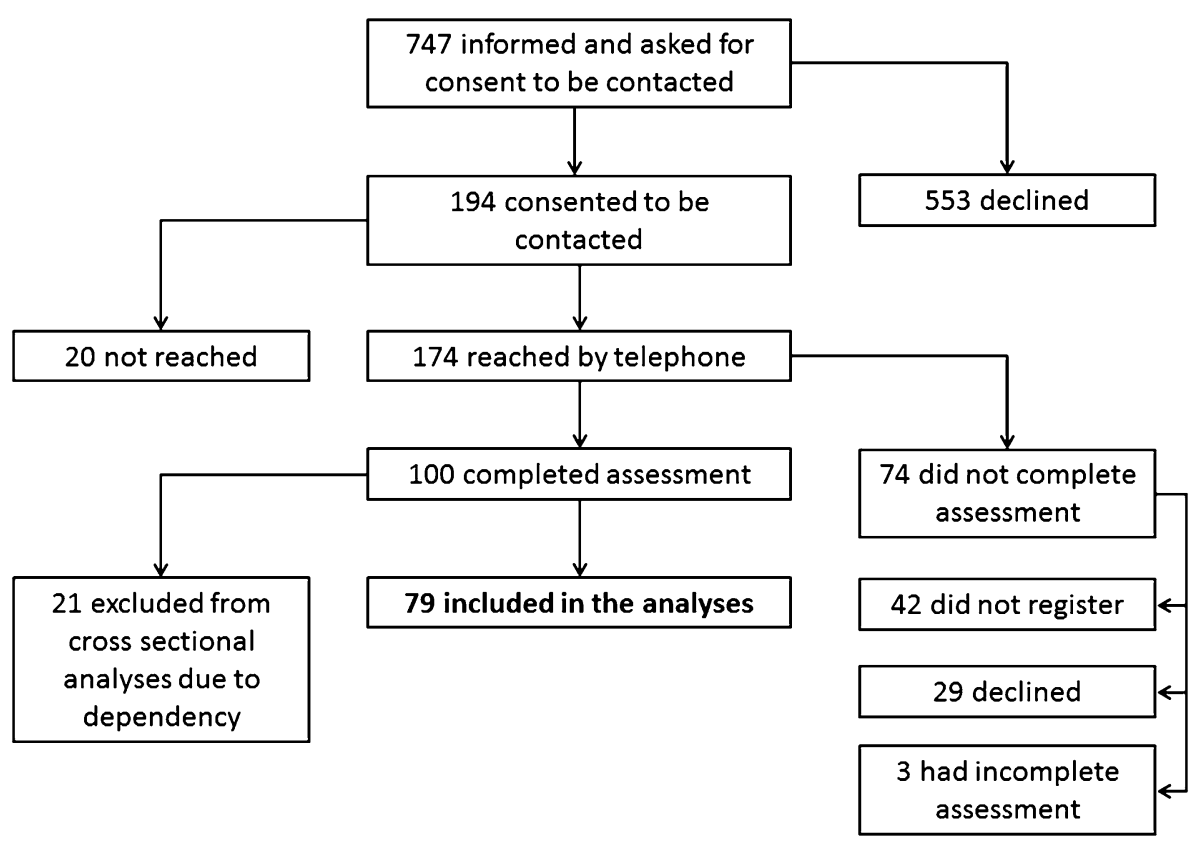

(8\%) with lymphoma, $10(13 \%)$ with a CNS-tumor, and $10(13 \%)$ with another malignancy.

\section{Measures}

\section{PTSD-Checklist Civilian Version}

Posttraumatic stress symptoms related to the child's cancer was assessed with the PCL-C (Weathers et al., 1993). The PCL-C consists of 17 items rated on a 5-point scale ranging from $1=$ Not at all to $5=$ Extremely, corresponding to the items assessing the $\mathrm{B}, \mathrm{C}$, and $\mathrm{D}$ criteria in the DSM-IV. The instructions are: "Below is a list of problems and complaints that parents of children with cancer sometimes have. Please read each one carefully and circle how much you have been bothered by that problem in the last month." Examples of items are: "Repeated, disturbing memories, thoughts, or images of a stressful experience related to your child's cancer disease?" and "Feeling emotionally numb or being unable to have loving feelings for those close to you?" The PCL-C was translated into Swedish using a forward-backward procedure following the guidelines of the EORTC Quality of Life Study Group (Koller et al., 2007). Ruggiero, Ben, Scotti, and Rabalais (2003) report that the instrument has adequate internal consistency, testretest reliability, and evidence for convergent and discriminant validity when compared to other well-established measures of PTSS, depression, and general anxiety. A value of 44 or above on the full scale suggests a diagnosis of PTSD (Blanchard, Jones-Alexander, Buckley, \& Forneris, 1996). Cronbach's $\alpha$ in the current sample was .92 .

\section{Beck Depression Inventory-II}

Symptoms of depression were assessed with the Beck Depression Inventory-II (BDI-II; Beck, Steer, \& Brown, 1996) consisting of 21 items rated on a 4-point scale ranging from 0 to 3 where each item had a list of four statements for each scale level. Examples of item statements are: $0=I$ do not feel sad, $1=I$ feel sad, $2=I$ am sad all the time and I can't snap out of it, $3=$ I am so sad or unhappy that I can't stand it. The BDI-II has shown good concurrent validity with its precursor BDI and the Hamilton Psychiatric Rating Scale. The suggested cut-offs are: 0-13 indicating minimal, 14-19 mild, 20-28 moderate, and 29-63 severe depression. Cronbach's $\alpha$ in the current sample was .92.

\section{Beck Anxiety Inventory}

Symptoms of anxiety was assessed with the Beck Anxiety Inventory (BAI; Beck, Epstein, Brown, \& Steer, 1988) consisting of 21 items rated on a 4-point scale ranging from $0=$ Not at all to $3=$ Severely. Instructions are: Below is a list of common symptoms of anxiety. Please carefully read each item in the list. Indicate how much you have been bothered by that symptom during the past month, including today, by circling the number in the corresponding space in the column next to each symptom. Examples of items are: "Numbness or tingling" and "Heart pounding or racing". The BAI has shown good test-retest reliability and convergent validity. The suggested cut-offs are: $0-7$ indicating 
minimal, 8-15 mild, 16-23 moderate, and 24-63 severe anxiety. Cronbach's $\alpha$ in the current sample was .89 .

\section{Acceptance and Action Questionnaire-II}

EA was assessed with the Acceptance and Action Questionnaire-II (AAQ-II; Bond et al., 2011). The original AAQ-II consisted of 10 items rated on a 7-point scale ranging from $1=$ Never true to $7=$ Always true. Examples of items are: "I am afraid of my feelings" and "Emotions cause problems in my life". A 7-item version (excluding items 1, 6, and 10) has shown the best psychometric properties with satisfactory test-retest reliability and construct validity (Bond et al., 2011) and was used in the current study. Total possible scores ranged from a low of 7 to a high of 49, with higher scores indicating more EA. Cronbach's $\alpha$ of the 7-item version in the current sample was .89 .

\section{Rumination Subscale of the Responses to Intrusions Questionnaire}

Rumination was assessed with the rumination subscale from the Responses to Intrusions Questionnaire (RIQrum). The subscale consisted of eight items rated on a 4-point scale ranging from $0=$ Never to $3=$ Always. Examples of items are "I think about why the event happened to me" and "I dwell on how I used to be before the event." The complete scale has shown adequate reliability and predictive validity (Clohessy \& Ehlers, 1999; Murray et al., 2002). The highest possible score on the rumination subscale is 24 and a higher score indicates more rumination. The RIQ was translated into Swedish using a forwardbackward procedure following the guidelines of the EORTC Quality of Life Study Group (Koller et al., 2007). Cronbach's $\alpha$ in the current sample was .86 .

\section{Statistical Analyses}

Correlations were used to investigate the relationships among study variables including age and gender for parents and children; diagnosis (leukemia vs. other diagnoses) and time since diagnosis for children; and level of education, employment status, marital status, and potential experience of previous trauma for parents. Categorical demographic variables were coded as dummy variables. Due to the small sample size and heterogeneity in terms of diagnosis the sample was grouped according to the most common diagnosis (leukemia) vs. the other diagnostic categories. Hierarchical multiple regression analyses were used to investigate the incremental explained variance in PTSS and symptoms of depression according to the hypotheses. Standardized regression coefficients $\beta$ were used to determine the relative contribution of these variables. All analyses were conducted in IBM SPSS Statistics $20{ }^{\circ}$. Three participants had missing data on age and two on time since diagnosis. Two participants had missing data on the RIQrum.

\section{Results}

Descriptive statistics and correlations between the main study variables are presented in Table 1. On the PCL-C, $48 \%$ of the participants scored above the suggested clinical cut-off. On the BDI-II, $35 \%$ of the participants scored above the cut-off indicating a moderate level of depression. On the BAI, $28 \%$ of the participants scored above the cutoff indicating moderate anxiety. As shown in Table 1, there were positive correlations between all study variables. As hypothesized, AAQ-II and RIQrum were positively associated with PCL-C, BDI-II, and BAI.

In order to determine if there were potential covariates that should be included in the main regression analyses, demographical variables were included in univariate correlation analyses together with the two dependent variables PCL-C and BDI-II. The results from these correlation analyses are presented in Table 2. The relationship of categorical variables such as gender, education, employment, marital status, child gender, and child's diagnosis to the PCL-C and BDI-II was assessed by point biserial correlation coefficients. As is evident, education level was related to both the PCL-C and the BDI-II, i.e., in comparison with the reference category of having university or college education (scored as zero), parents having 9 years or less of education (scored as 1) had higher scores on both instruments, i.e., parents with lower education had higher PTSS and depression symptoms than parents with more education. Marital status and employment status were also

Table 1 Descriptive statistics and Pearson's correlations between study variables

\begin{tabular}{lllllll}
\hline Variable & BDI-II & BAI & AAQ-II & RIQrum & $M$ & SD \\
\hline PCL-C & $.73 * * *$ & $.74 * * *$ & $.61^{* * *}$ & $.54^{\dagger * * *}$ & 43.5 & 13.6 \\
BDI-II & & $.70^{* * *}$ & $.72^{* * *}$ & $.64^{\dagger * * *}$ & 18.1 & 9.9 \\
BAI & & & $.61^{* * *}$ & $.53^{\dagger * *}$ & 12.4 & 8.0 \\
AAQ-II & & & & $.53^{\dagger * * *}$ & 20.0 & 8.0 \\
RIQrum & & & & & $7.3^{\dagger}$ & $4.8^{\dagger}$ \\
\hline
\end{tabular}

$n=79$. PCL-C PTSD-Checklist Civilian Version, BDI-II Beck Depression Inventory-II, $B A I$ Beck Anxiety Inventory, $A A Q-I I$ Acceptance and Action Questionnaire-II, RIQrum rumination subscale of the Responses to Intrusions Questionnaire

$\dagger \mathrm{n}=77$

$* * p<.01$

$* * * p<.001$ 

demographic characteristics and PTSS and symptoms of depression
Table 2 Correlations between

\begin{tabular}{|c|c|c|}
\hline Variable & PCL-C & BDI-II \\
\hline Age & -.20 & -.17 \\
\hline \multicolumn{3}{|l|}{ Gender } \\
\hline Father (reference) & - & - \\
\hline Mother & .18 & .18 \\
\hline \multicolumn{3}{|l|}{ Education level } \\
\hline University (reference) & - & - \\
\hline 12 years of education & -.14 & -.14 \\
\hline$\leq 9$ years of education & $.33 * *$ & $.31 * *$ \\
\hline \multicolumn{3}{|l|}{ Employment status } \\
\hline Employed (reference) & - & - \\
\hline Unemployed & .21 & $.40 * *$ \\
\hline Sick-leave & .17 & .11 \\
\hline \multicolumn{3}{|l|}{ Marital status } \\
\hline Living with child's other biological parent (reference) & - & - \\
\hline Living with child's stepparent & .09 & $.28 *$ \\
\hline Living alone & -.10 & -.20 \\
\hline Experience of previous trauma & -.10 & -.12 \\
\hline Time since child's diagnosis & .06 & .07 \\
\hline \multicolumn{3}{|l|}{ Child's gender } \\
\hline Boy (reference) & - & - \\
\hline Girl & -.10 & -.10 \\
\hline Child's age & -.11 & .04 \\
\hline \multicolumn{3}{|l|}{ Child's diagnosis } \\
\hline Leukemia (reference) & - & - \\
\hline Other diagnosis & -.01 & -.09 \\
\hline
\end{tabular}

$P C L-C$ PTSD-Checklist Civilian Version, BDI-II Beck Depression Inventory-II

$* p<.05$

$* * p<.01$

related to the BDI-II. In comparison with the reference category biological parent living with child's other biological parent (scored as 0 ), a biological parent living with a child's stepparent (scored as 1) tended to have a higher score on the BDI-II, i.e., was likely to report more depressive symptoms. In addition, being unemployed (scored as 1) was also associated with a higher score on the BDI-II, i.e., with more depressive symptoms, when compared with the reference category of being employed (scored as 0).

Results from the hierarchical multiple regression analysis with PCL-C as the dependent variable are presented in Table 3. In Step 1, parent level of education and BAI were included as predictors resulting in a significant overall model, $F(2,74)=48.61, p<.001$, that accounted for $57 \%$ of the variance in PCL-C. BAI was the only contributor to PCL-C in this initial step. In Step 2, BDI-II was added, which increased the model's explanatory power, $\Delta F(1,73)=15.93, p<.001$, by accounting for an additional $8 \%$ of the variance in PCL-C. Table 3 shows that BDI-II contributed to this model after accounting for education level and BAI. AAQ-II and RIQrum were added in Step 3 but resulted only in a non-significant change in model explanation, $\Delta F(2,71)=0.53, p=.59$. Table 3 shows that neither AAQ-II nor RIQrum contributed to this model after accounting for education level, BAI, and BDIII. Overall, AAQ-II and RIQrum did not provide incremental explanation of the variance in PCL-C, over and above the variance accounted for by the variables already included in the model. Inspection of the variance inflation factors (VIF) revealed no indication of multi-collinearity for the hierarchical regression models (VIF's ranged from between 1.08 and 3.12).

Results from the hierarchical multiple regression analysis with BDI-II as the dependent variable are presented in Table 4. In step 1, education level, employment status, marital status, and BAI were included as predictors resulting in a significant overall model, $F(4,72)=20.12$, $p<.001$, accounting for $53 \%$ of the variance in BDI-II. BAI was the only significant contributor to BDI-II in this step. In Step 2, PCL-C was added to the equation which resulted in an increase in model explanation, $\Delta F$ 
Table 3 Hierarchical multiple regression with PCL-C as dependent variable

\begin{tabular}{llllll}
\hline Variable & $B$ & SE & $\beta$ & $R_{\text {adj }}^{2}$ & $\Delta R^{2}$ \\
\hline$P C L-C$ & & & & & \\
Step 1 & & & & .56 & $.57 * * *$ \\
$\quad$ Education $^{\mathrm{a}}$ & 7.70 & 4.40 & 0.14 & & \\
BAI & 1.21 & 0.14 & $0.70^{* * *}$ & & \\
Step 2 & & & & .63 & $.08 * * *$ \\
$\quad$ Education $^{\mathrm{a}}$ & 4.92 & 4.01 & 0.09 & & \\
BAI & 0.76 & 0.17 & $0.44^{* * *}$ & & \\
BDI-II & 0.54 & 0.14 & $0.40^{* * *}$ & & \\
Step 3 & & & & .63 & .01 \\
Education & & & & \\
BAI & 5.01 & 4.10 & 0.09 & & \\
BDI-II & 0.71 & 0.17 & $0.41^{* * *}$ & & \\
AAQ-II & 0.44 & 0.17 & $0.32^{*}$ & & \\
RIQrum & 0.11 & 0.18 & 0.07 & & \\
\hline
\end{tabular}

$\overline{n=77 . \quad P C L-C \text { PTSD-Checklist Civilian Version, BDI-II Beck }}$ Depression Inventory-II, $B A I$ Beck Anxiety Inventory, $A A Q-I I$ Acceptance and Action Questionnaire-II, RIQrum rumination subscale of the Responses to Intrusions Questionnaire

$* * * p<.001$

${ }^{\text {a }}$ Up to 9 years of education versus university or college education

$(1,71)=20.08, p<.001$, accounting for an additional $10 \%$ of the variance in BDI-II. Table 4 indicates that PTSS contributed to this model after accounting for demographic variables and anxiety. AAQ-II and RIQrum were added in Step 3 resulting in a further increase in model explanation, $\Delta F(2,69)=12.93, \quad p<.001$, accounting for an additional $10 \%$ of the variance in BDIII. Table 4 indicates that both AAQ-II and RIQrum were significant predictors in this model after accounting for demographic variables, BAI, and PCL-C, with AAQ-II being a slightly stronger predictor. Overall, AAQ-II and RIQrum provided incremental explanation of the variance in BDI-II, over and above the variance accounted for by the variables already included in the model. Inspection of the VIFs revealed no indication of multi-collinearity for the hierarchical regression models (VIFs ranged from 1.17 to 2.73).

\section{Discussion}

The purpose was to investigate potential relationships between EA, rumination, PTSS, and symptoms of depression in parents of children on cancer treatment. As hypothesized, EA and rumination were positively associated with PTSS, symptoms of depression, and symptoms of
Table 4 Hierarchical multiple regression with BDI-II as dependent variable

\begin{tabular}{|c|c|c|c|c|c|}
\hline Variable & $B$ & $S E$ & $\beta$ & $R_{\text {adj }}^{2}$ & $\Delta R^{2}$ \\
\hline \multicolumn{6}{|l|}{$B D I-I I$} \\
\hline Step 1 & & & & .50 & $.53 * * *$ \\
\hline Education $^{\mathrm{a}}$ & 3.19 & 3.52 & 0.08 & & \\
\hline Employment status ${ }^{\mathrm{b}}$ & 4.41 & 3.87 & 0.12 & & \\
\hline Marital status ${ }^{\mathrm{c}}$ & 2.53 & 3.46 & 0.07 & & \\
\hline BAI & 0.78 & 0.11 & $0.62 * * *$ & & \\
\hline Step 2 & & & & .61 & $.10 * * *$ \\
\hline Education $^{\mathrm{a}}$ & -0.11 & 3.22 & -0.01 & & \\
\hline Employment status ${ }^{\mathrm{b}}$ & 5.48 & 3.45 & 0.15 & & \\
\hline Marital status ${ }^{\mathrm{c}}$ & 3.39 & 3.08 & 0.10 & & \\
\hline BAI & 0.33 & 0.14 & $0.26^{*}$ & & \\
\hline PCL-C & 0.36 & 0.08 & $0.50 * * *$ & & \\
\hline Step 3 & & & & .71 & $.10 * * *$ \\
\hline Education $^{\mathrm{a}}$ & 0.62 & 2.79 & 0.02 & & \\
\hline Employment status ${ }^{\mathrm{b}}$ & 6.56 & 3.00 & 0.18 & & \\
\hline Marital status ${ }^{\mathrm{c}}$ & -0.16 & 2.76 & -0.01 & & \\
\hline BAI & 0.13 & 0.13 & 0.11 & & \\
\hline PCL-C & 0.22 & 0.08 & $0.30 * *$ & & \\
\hline AAQ-II & 0.39 & 0.11 & $0.32 * * *$ & & \\
\hline RIQrum & 0.43 & 0.16 & $0.21 * *$ & & \\
\hline
\end{tabular}

$n=77$. PCL-C PTSD-Checklist Civilian Version, BDI-II Beck Depression Inventory-II, $B A I$ Beck Anxiety Inventory, $A A Q-I I$ Acceptance and Action Questionnaire-II, RIQrum rumination subscale of the Responses to Intrusions Questionnaire

$* p<.05$

$* * p<.01$

$* * * p<.001$

${ }^{\text {a }}$ Up to 9 years of education versus university or college education

b Being unemployed versus being employed

c Living with child's stepparent versus living with child's biological parent

anxiety. Also as hypothesized, EA and rumination accounted for incremental explained variance in symptoms of depression over and above that explained by PTSS, when controlling for symptoms of anxiety and demographic characteristics. Based on previous research we expected the contribution of EA and rumination in PTSS to be less pronounced compared to their contribution in symptoms of depression, but still significant. However, neither EA nor rumination accounted for incremental explained variance in PTSS when controlling for symptoms of anxiety, symptoms of depression, and demographic characteristics.

There is a growing literature showing that EA is a core process in psychological distress and general adjustment (Chawla \& Ostafin, 2007; Hayes et al., 1996), and that 
rumination is related to maladjustment after trauma (Ehring, Ehlers, \& Glucksman, 2008a). The findings suggest that EA and rumination could be problematic processes in parents of children on cancer treatment, particularly with regard to depression. This also agrees with findings in parents in other pediatric populations. Greco et al. (2005) found that EA correlated with PTSS and parenting stress in mothers of pre-term born infants. To our knowledge, the current study is the first to investigate the role of EA and rumination and their potential relationship with PTSS and symptoms of depression in parents of children on cancer treatment. Findings from the correlation analyses suggest that parents who are more inclined to avoid distressing thoughts and feelings and who have difficulties taking action in the presence of such experiences, and who engage in repetitive thinking when reminded of their child's disease, endorse PTSS and symptoms of depression to a greater extent.

Of interest is the finding that the relationship of EA and rumination to PTSS is different from their relationship to depression, which supports previous research in various populations (Plumb et al., 2004; Tull et al., 2004). This finding indicates that EA and rumination are associated with symptoms of depression when the influence of PTSS is controlled for, but EA and rumination are not associated with PTSS when symptoms of depression are controlled for. The finding further indicates that the associations between EA, rumination, and PTSS may in part be due to their shared association with symptoms of depression. It may be that EA is more closely related to the general distress of depression than to the more specific type of distress of PTSS. Spinhoven et al., (2014) showed that EA is a predictor of emotional disorders and the current results indicate that the relationship between EA and depression might be stronger than the relationship between EA and PTSS. This would also be in line with the notion of EA as a broad behavioral construct underlying general psychological distress. Morina (2011) showed that both EA and rumination were associated to PTSS and depression in widows who lost their husband in the Kosovo war, however, the analyses did not adjust for the shared variance between PTSS and depression.

Repetitive abstract thinking such as rumination has received increasing interest as a contributor to states of psychological distress such as depression and PTSS (Ehring, Frank, \& Ehlers, 2008b; Murray et al., 2002; NolenHoeksema, Wisco, \& Lyubomirsky, 2008). Rumination has been suggested to serve the function of avoiding processing of negative emotion (Smith \& Alloy, 2009) and may be a form of EA. In this study there was a substantial correlation $(r=.51)$ between rumination and EA, suggesting that these processes are overlapping but also distinct. For parents of children on cancer treatment, it might also be the case that future-oriented repetitive abstract thinking, such as worry, may be more pronounced than rumination, which is past-oriented.

Some limitations to this study should be noted. Firstly, the data presented are cross-sectional which preclude conclusions regarding causality. PTSS and symptoms of depression were used as dependent variables and EA and rumination as predictors, but relationships may be reversed. Furthermore, the sample is small and potentially biased by self-selection. Participants were invited to take part in a RCT investigating the efficacy of an Internetbased guided self-help intervention. In the current sample, the mean level of PTSS was 43.5 ( $\mathrm{SD}=13.6)$, which is higher than was found in a previous Swedish cohort study of parents of children diagnosed with cancer, which also used the PCL-C, and for which the mean level of PTSS was 36.5 ( $\mathrm{SD}=12.1) 2$ months after diagnosis (Pöder et al., 2008). Furthermore, one could argue that the enrollment rate was poor, but it might also be that the enrollment rate adequately reflected the proportion of parents of children of children on cancer treatment who experience a need for psychological support. It has been shown that $28 \%$ of parents of children diagnosed with cancer report symptoms indicating potential PTSD 2 months after their child's diagnosis (Pöder et al., 2008) and that $46 \%$ report a need to meet with a psychologist at the same assessment (Pöder \& von Essen, 2009). In the current study, $26 \%$ of the approached parents expressed an interest in participating in the RCT and consented to be contacted. This figure might adequately reflect the proportion of parents who experienced a need for psychological help at the time when they were offered participation in the present study. However, as data were not collected from those declining participation we do not know whether consenting and non-consenting parents differed on key characteristics including psychological distress and need of psychological help. A proportion of those consenting failed to complete the assessment. Reasons for this were not investigated but one might speculate that parents of children on cancer treatment are in a stressful situation with many demands. Even though these parents might consent to participate in a research study, they still might have difficulty finding the time to participate. Furthermore, assessment was Internetbased requiring the parents to actively log into a web-portal which for some may have been a barrier.

Despite limitations, the current study has some implications for research and clinical practice. Findings suggest that rumination and EA in particular are important constructs for further study in parents of children on cancer treatment. However, the results need to be replicated and the temporal relationships between EA, rumination, PTSS, and symptoms of depression need to be investigated in larger samples. The results tentatively suggest that parents 
of children on cancer treatment who experience psychological distress might benefit from interventions targeting rumination and EA in particular. Such interventions may include training of skills fostering flexibility around and acceptance of distressing thoughts and feelings, and skills that reduce repetitive thinking. However, controlled applied research is needed to determine the efficacy and applicability of such interventions among parents of children on cancer treatment.

Acknowledgments This research was supported by grants from the Swedish Research Council (Grant Numbers K2008-70X-20836-01-3, K2011-70X-20836-04-4), the Swedish Cancer Society (Grant Number 2010/276), and the Swedish Childhood Cancer Foundation (Grant Number PROJ08/010, PRO12/028).

\section{Compliance with Ethical Standards}

Conflict of interest Martin Cernvall, Ellen Skogseid, Per Carlbring, Lisa Ljungman, Gustaf Ljungman, and Louise von Essen declared that they have no conflict of interest.

Human and Animal Rights and Informed Consent All procedures followed were in accordance with the ethical standards of the responsible committee on human experimentation (the Central Ethic Review Board in Sweden) and with the Helsinki Declaration of 1975, as revised in 2000. Informed consent was obtained for all participants for being included in this study.

Open Access This article is distributed under the terms of the Creative Commons Attribution 4.0 International License (http://crea tivecommons.org/licenses/by/4.0/), which permits unrestricted use, distribution, and reproduction in any medium, provided you give appropriate credit to the original author(s) and the source, provide a link to the Creative Commons license, and indicate if changes were made.

\section{References}

American Psychiatric Association. (2000). Diagnostic and statistical manual of mental disorders: DSM-IV-TR. Washington, DC: American Psychiatric Association.

American Psychiatric Association. (2013). American psychiatric association: Diagnostic and statistical manual of mental disorders (5th ed.). Arlington, VA: American Psychiatric Association.

Beck, A. T., Epstein, N., Brown, G., \& Steer, R. A. (1988). An inventory for measuring clinical anxiety: Psychometric properties. Journal of Consulting and Clinical Psychology, 56(6), 893-897.

Beck, A. T., Steer, R. A., \& Brown, G. K. (1996). Beck depression inventory-II. San Antonio, TX: The Psychological Corporation.

Blanchard, E. B., Jones-Alexander, J., Buckley, T. C., \& Forneris, C. A. (1996). Psychometric properties of the PTSD Checklist (PCL). Behaviour Research and Therapy, 34(8), 669-673.

Boman, K. K., Viksten, J., Kogner, P., \& Samuelsson, U. (2004). Serious illness in childhood: The different threats of cancer and diabetes from a parent perspective. The Journal of Pediatrics, 145(3), 373-379.

Bond, F. W., Hayes, S. C., Baer, R. A., Carpenter, K. M., Guenole, N., Orcutt, H. K., ... Zettle, R. D. (2011). Preliminary psychometric properties of the Acceptance and Action
Questionnaire-II: A revised measure of psychological inflexibility and experiential avoidance. Behavior Therapy, 42(4), 676-688. doi:10.1016/j.beth.2011.03.007.

Breslau, N., Lucia, V. C., \& Davis, G. C. (2004). Partial PTSD versus full PTSD: An empirical examination of associated impairment. Psychological Medicine, 34(7), 1205-1214. Retrieved from http://www.ncbi.nlm.nih.gov/pubmed/15697047.

Campbell-Sills, L., Barlow, D. H., Brown, T. A., \& Hofmann, S. G. (2006). Effects of suppression and acceptance on emotional responses of individuals with anxiety and mood disorders. Behaviour Research and Therapy, 44(9), 1251-1263. doi:10. 1016/j.brat.2005.10.001.

Cernvall, M., Carlbring, P., Ljungman, G., \& von Essen, L. (2013). Guided self-help as intervention for traumatic stress in parents of children with cancer: Conceptualization, intervention strategies, and a case study. Journal of Psychosocial Oncology, 31(1), 13-29. doi:10.1080/07347332.2012.741095.

Cernvall, M., Carlbring, P., Ljungman, L., Ljungman, G., \& von Essen, L. (2015). Internet-based guided self-help for parents of children on cancer treatment: A randomized controlled trial. Psycho-Oncology, 24(9), 1152-1158. doi:10.1002/pon.3788.

Chawla, N., \& Ostafin, B. (2007). Experiential avoidance as a functional dimensional approach to psychopathology: An empirical review. Journal of Clinical Psychology, 63(9), 871-889.

Clohessy, S., \& Ehlers, A. (1999). PTSD symptoms, response to intrusive memories and coping in ambulance service workers. British Journal of Clinical Psychology, 38(3), 251-265.

Dolgin, M. J., Phipps, S., Fairclough, D. L., Sahler, O. J. Z., Askins, M., Noll, R. B., ... Katz, E. R. (2007). Trajectories of adjustment in mothers of children with newly diagnosed cancer: A natural history investigation. Journal of Pediatric Psychology, 32(7), 771-782. doi:10.1093/jpepsy/jsm013.

Ehlers, A., Mayou, R. A., \& Bryant, B. (1998). Psychological predictors of chronic posttraumatic stress disorder after motor vehicle accidents. Journal of Abnormal Psychology, 107(3), 508-519.

Ehring, T., Ehlers, A., \& Glucksman, E. (2008a). Do cognitive models help in predicting the severity of posttraumatic stress disorder, phobia, and depression after motor vehicle accidents? A prospective longitudinal study. Journal of Consulting and Clinical Psychology, 76(2), 219-230.

Ehring, T., Frank, S., \& Ehlers, A. (2008b). The role of rumination and reduced concreteness in the maintenance of posttraumatic stress disorder and depression following trauma. Cognitive Therapy and Research, 32(4), 488-506.

Farmer, R. F., \& Chapman, A. L. (2008). Behavioral interventions in cognitive behavior therapy: Practical guidance for putting theory into action. Washington, DC: American Psychological Association.

Fledderus, M., Bohlmeijer, E. T., \& Pieterse, M. E. (2010). Does experiential avoidance mediate the effects of maladaptive coping styles on psychopathology and mental health? Behavior Modification, 34(6), 503-519. doi:10.1177/0145445510378379.

Greco, L. A., Heffner, M., Poe, S., Ritchie, S., Polak, M., \& Lynch, S. K. (2005). Maternal adjustment following preterm birth: Contributions of experiential avoidance. Behavior Therapy, 36(2), $177-184$.

Gross, J. J., \& Levenson, R. W. (1997). Hiding feelings: The acute effects of inhibiting negative and positive emotion. Journal of Abnormal Psychology, 106(1), 95-103.

Hayes, S. C., Strosahl, K. D., \& Wilson, K. G. (1999). Acceptance and commitment therapy: An experiential approach to behavior change. New York, NY: The Guilford Press.

Hayes, S. C., Wilson, K. G., Gifford, E. V., Follette, V. M., \& Strosahl, K. D. (1996). Experiential avoidance and behavioral disorders: A functional dimensional approach to diagnosis and 
treatment. Journal of Consulting and Clinical Psychology, 64(6), $1152-1168$.

Kazak, A. E., Boeving, C. A., Alderfer, M. A., Hwang, W. T., \& Reilly, A. (2005). Posttraumatic stress symptoms during treatment in parents of children with cancer. Journal of Clinical Oncology, 23(30), 7405-7410. doi:10.1200/JCO.2005.09.110.

Kenny, D. A. (2011). Commentary: Dyadic analyses of family data. Journal of Pediatric Psychology, 36(5), 630-633. doi:10.1093/ jpepsy/jsq124.

Koller, M., Aaronson, N. K., Blazeby, J., Bottomley, A., Dewolf, L., Fayers, P., ... West, K. (2007). Translation procedures for standardised quality of life questionnaires: The European Organisation for Research and Treatment of Cancer (EORTC) approach. European Journal of Cancer (Oxford, England : 1990), 43(12), 1810-20. doi:10.1016/j.ejca.2007.05.029.

Ljungman, L., Cernvall, M., Grönqvist, H., Ljótsson, B., Ljungman, G., \& von Essen, L. (2014). Long-term positive and negative psychological late effects for parents of childhood cancer survivors: A systematic review. PLoS One, 9(7), 103340. doi:10.1371/journal.pone.0103340.

Ljungman, L., Hovén, E., Ljungman, G., Cernvall, M., \& von Essen, L. (2015). Does time heal all wounds? A longitudinal study of the development of posttraumatic stress symptoms in parents of survivors of childhood cancer and bereaved parents. PsychoOncology. doi:10.1002/pon.3856.

Marx, B. P., \& Sloan, D. M. (2005). Peritraumatic dissociation and experiential avoidance as predictors of posttraumatic stress symptomatology. Behaviour Research and Therapy, 43(5), $569-583$.

Morina, N. (2011). Rumination and avoidance as predictors of prolonged grief, depression, and posttraumatic stress in female widowed survivors of war. Journal of Nervous and Mental Disease, 199(12), 921-927. doi:10.1097/NMD.0b013e31823 92aae.

Murray, J., Ehlers, A., \& Mayou, R. A. (2002). Dissociation and posttraumatic stress disorder: Two prospective studies of road traffic accident survivors. The British Journal of Psychiatry, 180(4), 363-368. doi:10.1192/bjp.180.4.363.

Nolen-Hoeksema, S., Wisco, B. E., \& Lyubomirsky, S. (2008). Rethinking rumination. Perspectives on Psychological Science, 3(5), 400-424. doi:10.1111/j.1745-6924.2008.00088.x.
Orcutt, H. K., Pickett, S. M., \& Pope, E. B. (2005). Experiential avoidance and forgiveness as mediators in the relation between traumatic interpersonal events and posttraumatic stress disorder symptoms. Journal of Social and Clinical Psychology, 24(7), 1003-1029.

Plumb, J. C., Orsillo, S. M., \& Luterek, J. A. (2004). A preliminary test of the role of experiential avoidance in post-event functioning. Journal of Behavior Therapy and Experimental Psychiatry, 35(3), 245-257.

Pöder, U., Ljungman, G., \& von Essen, L. (2008). Posttraumatic stress disorder among parents of children on cancer treatment: A longitudinal study. Psycho-Oncology, 17(5), 430-437. doi:10. 1002/pon. 1263.

Pöder, U., \& von Essen, L. (2009). Perceptions of support among Swedish parents of children on cancer treatment: A prospective, longitudinal study. European Journal of Cancer Care, 18(4), $350-357$.

Ruggiero, K. J., Ben, K. D., Scotti, J. R., \& Rabalais, A. E. (2003). Psychometric properties of the PTSD checklist-civilian version. Journal of Traumatic Stress, 16(5), 495-502.

Smith, J. M., \& Alloy, L. B. (2009). A roadmap to rumination: A review of the definition, assessment, and conceptualization of this multifaceted construct. Clinical Psychology Review, 29(2), 116-128. doi:10.1016/j.cpr.2008.10.003.

Spinhoven, P., Drost, J., de Rooij, M., van Hemert, A. M., \& Penninx, B. W. (2014). A longitudinal study of experiential avoidance in emotional disorders. Behavior Therapy, 45(6), 840-850. doi:10. 1016/j.beth.2014.07.001.

Tull, M. T., Gratz, K. L., Salters, K., \& Roemer, L. (2004). The role of experiential avoidance in posttraumatic stress symptoms and symptoms of depression, anxiety, and somatization. The Journal of Nervous and Mental Disease, 192(11), 754-761.

Wells, A. (2008). Metacognitive therapy for anxiety and depression. New York, NY: The Guilford Press.

Wenzlaff, R. M., \& Wegner, D. M. (2000). Thought suppression. Annual Review of Psychology, 51, 59-91. doi:10.1146/annurev. psych.51.1.59.

Weathers, F. W., Litz, B. T., Herman, D. S., Huska, J. A., \& Keane, T. M. (1993). The PTSD checklist (PCL): Reliability, validity, and diagnostic utility. In The Annual Meeting of International Society for Traumatic Stress Studies. San Antonio, TX. 